\title{
An enhanced model for testing asynchronous communicating systems
}

\author{
Myungchul Kim, Jaehwi Shin ${ }^{+}$, Samuel T. Chanson", and Sungwon Kang ${ }^{+}$ \\ Information and Communications University \\ Yousonggu, Taejon, Korea 305-348 \\ mckim@icu.ac.kr \\ ${ }^{+}$Korea Telecom $R \&$ D Group \\ Sochogu, Seoul, Korea 137-792 \\ \{jhshin,kangsw\}@sava.kotel.co.kr \\ "Hong Kong University of Science and Technology \\ Clear Water Bay, Kowloon, Hong Kong \\ chanson@cs.ust.hk
}

\begin{abstract}
This paper studies the problem of testing concurrent systems considered as blackboxes and specified using asynchronous Communicating Finite State Machines. In our previous work [Kim 96], an approach to derive test cases for concurrent systems was developed. The approach addressed the state space explosion problem by introducing a causality relation model and the concept of logical time to express true concurrency. This paper builds on and extends the previous work. The model is enhanced to improve the quality (i.e., verdict capability) of test cases by relaxing the unit-time assumption to any natural number and by presenting a computationally efficient algorithm for deriving the test cases. Finally the approach is generalized to describe timing constraints with any real numbers.
\end{abstract}

\section{Keywords}

Protocol Testing, Concurrent Systems, Logical Time, Conformance Testing, Asynchronous Communicating Finite State Machines. 


\section{INTRODUCTION}

In order to specify precisely the concurrent behavior of communication protocols and services in a distributed system, formal description techniques such as Communicating Finite State Machines (CFSMs), Estelle, SDL and LOTOS have been developed. These techniques allow interactions among multiple machines (or modules or processes) in a concurrent system to be specified formally. To increase the confidence in protocol products implemented according to international standards, various protocol testing methodologies have been developed and are being used.

Until recently, most work on automatic test case generation has focused on single machine specifications even though the implementation may consist of multiple machines. Typically, a preprocessing phase is used to translate the multiple machine specifications into a single system specification in order to take advantage of the many existing testing methods for sequential systems. Usually reachability analysis is used in the translation process. Unfortunately, the "state space explosion" problem is often encountered during reachability analysis. In addition, it is difficult to express timing constraints using reachability analysis techniques [West 78].

To alleviate the state space explosion problem, the concept of "true concurrency" or "simultaneous execution of events" has been introduced by several researchers to reduce the number and lengths of test sequences in the test suite for concurrent systems (see for example [West 78, Itoh 83, Kajiw 85, Araka 91]). The concept works because a single event sequence using true concurrency could represent many interleaved event sequences. These methods treat concurrent systems as blackboxes. The control flow graph representing the global behavior of a given concurrent system is first constructed and conventional test case generation methods are applied to the control flow graph. To reduce the number and the lengths of test sequences, the notion of so-called reduced implementation sequences is used based on true concurrency. However, the methods for deriving the reduced implementation sequences are usually based on heuristics and are often informally described. Another aspect worth noting is that the reduced implementation sequences may reduce the verdict capability (i.e., may lead to higher probability of inconclusive verdicts).

We have presented a formal methodology of generating test sequences using true concurrency and logical time [Lampo 78, Rayna 92] for concurrent systems described as asynchronous CFSMs [Kim 96]. The resulting test sequence was called Minimal Causality Path (MCP) calculated based on the assumption that the duration of each event is exactly one unit time. In fact, the 'MCP' and 'reduced implementation sequence' turn out to be the same test sequence although the procedures for obtaining them are different. However 
as a result of the unit-time assumption, tests using MCPs have low verdict capabilities.

In this paper we propose to enhance the verdict capability by allowing more state space to be investigated. There is a tradeoff between the verdict capability and size of the state space explored. The first enhancement is to relax the unit-time assumption on event duration to any arbitrary integer value and present a computationally efficient method for deriving the test cases. Then we show how the approach can be generalized to describe timing constraints using a causal relation model and real time logical clock.

The rest of the paper is organized as follows: Section 2 surveys some related work in protocol testing, and Section 3 summarizes the model proposed in [Kim 96] and discusses the verdict capability of the resultant test cases. Section 4 provides an enhancement to the model by relaxing the assumption of unit time to any natural number. In addition, two fundamental properties on the event sequence of concurrent systems are presented, and a computationally efficient algorithm to deriving test cases under the relaxed logical time framework is derived based on the two properties. In Section 5, the model is generalized to express timing constraints by any real value. Finally Section 6 concludes the paper.

\section{RELATED WORK}

According to the ISO conformance testing methodology, an Implementation Under Test (IUT) is viewed as a blackbox for testing purposes [ISO 94]. Our work aims at testing asynchronous CFSMs which are treated as blackboxes. By comparison, most of the existing work in software testing such as [Young 88, Taylor 92, Tai 95] is based on synchronous communicating programs where the internal code is assumed to be accessible (the white-box approach). Since our work is focused on protocol testing, we present below a brief survey of some related papers on testing protocols modeled as concurrent systems.

In [Lee 93], an approach for conformance testing of protocols specified as a collection of synchronous CFSMs is presented. The approach consists of two parts: pruning and a guided random walk procedure. This allows some parts of the behavior space to be removed from consideration. Also, the model is based on interleaving in order to explore the state space.

Cavalli et al. [Caval 93] present a test sequence generation method based on Unique Input/Output sequences and signatures in a framework which allows conformance test sequences to be obtained from LOTOS specifications. The model is for synchronous communicating systems only. 
Luo et al. [Luo 94] present a model of generating test sequences for concurrent systems and communication protocol modeled as communicating nondeterministic finite state machines (CNFSMs). After the CNFSMs are transformed into a single nondeterministic finite state machine (NFSM) by reachability analysis, test sequences are obtained from the resulting NFSM using the generalized Wp-method. The approach gives rise to the state space explosion problem easily because the reachability analysis in the transformation phase.

In [Ulric 95], an approach to derive a concurrent transition tour as a test suite from multi-module specifications described by a restricted class of Calculus of Communicating Systems is presented. This approach alleviates the state space explosion problem by introducing a true concurrency model. The model is for synchronous communicating systems and uses some heuristics in constructing the order of messages.

[Henni 97] proposes an approach for generating test cases in Concurrent TTCN from a system of asynchronous communicating finite state machines. An algorithm is given for generating a noninterleaving model of prime event structures (adopted from verification and concurrency theory) from a generalized model of asynchronous communicating finite state machines. The work also deals with test case generation from the prime event structure. However, computational efficiency, verdict capability and timing constraints have not been considered.

Ulric et al. [Ulric 97] presents a concurrency model, called behavior machine, and its construction algorithm. Further the paper outlines how test derivation can be based on the new concurrency model in order to derive test suites that still exhibit true concurrency between test events. The model is for synchronous communicating systems and uses the Petri net unfolding algorithm.

Our work aims at testing asynchronous CFSMs treated as blackboxes, and is a non-heuristic (i.e., exact) approach using a true concurrency model. The model is evaluated in terms of computational efficiency and verdict capability, and generalized to describe timing constraints using a causal relation model and real time logical clock to increase the verdict capability of the test cases.

\section{BACKGROUND}

In this Section we introduce the concept of logical clocks and summarize our previous work. 


\subsection{Logical clocks}

Lamport showed that asynchronous distributed executions can be characterized by a partial order relation on the events produced. The relation, called the causality relation (or happened before relation) is denoted by $\rightarrow$ [Lampo 78]. Let $\mathrm{E}$ be a set of events produced by the execution of a distributed system. For $e, e^{\prime}, e^{\prime \prime} \in E, e \rightarrow e^{\prime}$ holds if:

1. e and $\mathrm{e}^{\prime}$ are events in the same process and e precedes $\mathrm{e}^{\prime}$,

2. e is a send event and $e^{\prime}$ is the corresponding receive event, or

3. there exists $\mathrm{e}^{\prime \prime}$ such that $\mathrm{e} \rightarrow \mathrm{e}^{\prime \prime}$ and $\mathrm{e}^{\prime \prime} \rightarrow \mathrm{e}^{\prime}$.

Two events $\mathrm{e}$ and $\mathrm{e}^{\prime}$ are said to be causally related if $\mathrm{e} \rightarrow \mathrm{e}^{\prime}$ or $\mathrm{e}^{\prime} \rightarrow \mathrm{e}$ holds. If neither $\mathrm{e} \rightarrow \mathrm{e}^{\prime}$ nor $\mathrm{e}^{\prime} \rightarrow \mathrm{e}$ holds, these events are concurrent (or mutually independent), denoted by $\mathrm{e} \| \mathrm{e}^{\prime}$.

According to the above principles, a number of time stamping mechanisms have been devised including linear time, vector time, and matrix time [Rayna 92]. They are important in studying the behavior or distributed systems. The linear time $c_{i}$ associated with process $P_{i}$, for example, can be computed as follows (all values of $c_{i}$ 's are initialized to zero):

- When $P_{i}$ executes an internal event or a send event, the time is updated, i.e., $\mathrm{c}_{\mathrm{i}}=\mathrm{c}_{\mathrm{i}}+\mathrm{d}$, where $d$ represents the duration of the corresponding operation. In the case of a send event, the message carries the updated $c_{i}$ value as the timestamp.

- When $P_{\mathrm{i}}$ executes a receive event where the message contains a timestamp $t s$, the time is computed as $\mathrm{c}_{\mathrm{i}}=\max \left(\mathrm{c}_{\mathrm{i}}, \mathrm{ts}\right)+\mathrm{d}$.

\subsection{Overview of our basic framework [Kim 96]}

A concurrent system consists of a number of sequential systems. Each sequential system is modeled as a Finite State Machine (FSM). It is assumed that there is no global physical clock; that is, the system model is asynchronous. Thus, the concurrent system is represented by asynchronous CFSMs and treated as blackboxes. The environment (e.g., operators, testers, and/or subsystems which are not under test) of a concurrent system is also modeled as FSMs. The FSMs communicate with each other by messages and are assumed to contain no message loops. The underlying message passing system provides asynchronous communication and is assumed to be reliable; that is, there is no message loss, distortion or duplication. An event is the atomic execution of an operation within a system. 
We assume that a concurrent system consists of subsystems which do not share variables; instead, they interact by message passing. Thus, there are no dataraces in this model. Data races occur if concurrent events have read/write or write/write access conflicts to shared variables. Since each subsystem is treated as a blackbox, only communication events such as message send and receive events are considered.

Definition 1: A Global Event (GE) is defined by the set of all (observable) events that occur at the same time in all the FSMs of a concurrent system. Namely, GE $=\left(e_{1}, \ldots, e_{i}, \ldots, e_{n}\right)$ where $e_{i}$ is an event of the $i$-th FSM.

Note that since we are interested in blackbox testing, GE does not include internal events. The GE consists of the send and receive events of the subsystems and expresses true concurrency.

A path is a sequence of events performed by an FSM starting and ending in the same initial state and is denoted as $\mathrm{e}^{1} \rightarrow \mathrm{e}^{2} \rightarrow \ldots \rightarrow \mathrm{e}^{\mathrm{m}}$. We consider, for simplicity, the second visit to the initial state of an FSM as termination. This is not a limitation since subsequent behavior (if any) can be viewed as another path.

Definition 2: Paths[i] is the set of all event sequences that can be performed by the i-th FSM, i.e., Paths[i] $=\{\operatorname{Path}(i, 1), \operatorname{Path}(i, 2), \ldots\}$.

For the example in Figure 1 adopted from [Kajiw 85], the Paths[i] are given below:

Paths[1] $=\{-\mathrm{a} \rightarrow-\mathrm{b} \rightarrow+\mathrm{c} \rightarrow-\mathrm{d} \rightarrow+\mathrm{f},-\mathrm{a} \rightarrow-\mathrm{b} \rightarrow-\mathrm{c} \rightarrow+\mathrm{f}\}$

Paths[2] $=\{+\mathrm{a} \rightarrow+\mathrm{d} \rightarrow-\mathrm{f} \rightarrow-\mathrm{g},+\mathrm{a} \rightarrow+\mathrm{e} \rightarrow-\mathrm{f} \rightarrow-\mathrm{g}\}$

Paths[3] $=\{+\mathrm{b} \rightarrow+\mathrm{c} \rightarrow-\mathrm{e} \rightarrow+\mathrm{g},+\mathrm{b} \rightarrow-\mathrm{c} \rightarrow+\mathrm{g}\}$

From all the possible paths of the $n$ FSMs, we construct path vectors each of which represents a situation where the FSMs are communicating with one another (See Definition 3).

Definition 3: A Concurrent Path (CP) is a vector of event sequences, denoted by $\mathrm{CP}_{\mathrm{k}}=\left\langle\right.$ Path $\left(1,1_{\mathrm{k}}\right), \ldots$, Path $\left(\mathrm{i}, \mathrm{i}_{\mathrm{k}}\right), \ldots$, Path $\left.\left(\mathrm{n}, \mathrm{n}_{\mathrm{k}}\right)\right\rangle . \mathrm{CP}_{\mathrm{k}}$ is an element of Paths[1] $\times \ldots \times$ Paths[i] $\times \ldots \times$ Paths[n] where the operator ' $\times$ ' is the Cartesian product such that a send event is matched with the corresponding receive event in chronological order. An element Path $\left(i, i_{k}\right)$ of $\mathrm{CP}_{k}$ is null if the $i$-th FSM is idle. If a send event does not match with the corresponding receive event during generating $\mathrm{CP}_{k}$, event sequences already generated for the $\mathrm{CP}_{k}$ is kept. 

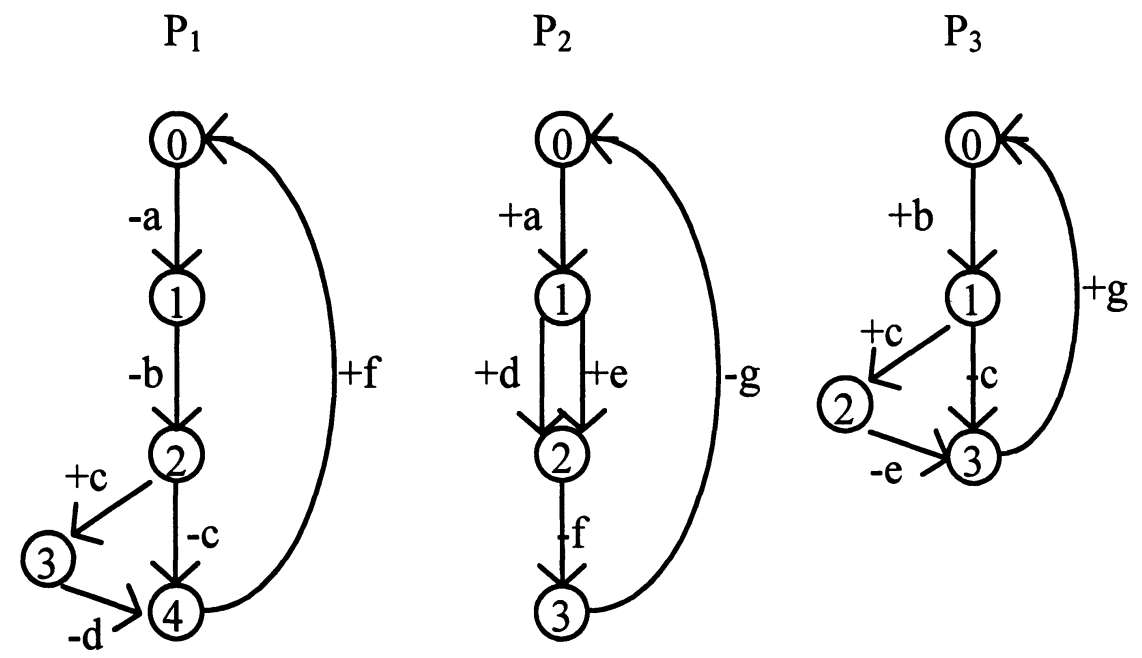

Figure 1. Example of a concurrent system

Definition 3 is illustrated using the example in Figure 1. Here, we have 6 different CP's as follows:

$$
\begin{aligned}
& \mathrm{CP}_{1}=<-\mathrm{a} \rightarrow-\mathrm{b} \rightarrow+\mathrm{c} \rightarrow-\mathrm{d} \rightarrow+\mathrm{f},+\mathrm{a} \rightarrow+\mathrm{d} \rightarrow-\mathrm{f} \rightarrow-\mathrm{g},+\mathrm{b} \rightarrow-\mathrm{c} \rightarrow+\mathrm{g}> \\
& \mathrm{CP}_{2}=<-\mathrm{a} \rightarrow-\mathrm{b} \rightarrow-\mathrm{c} \rightarrow+\mathrm{f},+\mathrm{a} \rightarrow+\mathrm{e} \rightarrow-\mathrm{f} \rightarrow-\mathrm{g},+\mathrm{b} \rightarrow+\mathrm{c} \rightarrow-\mathrm{e} \rightarrow+\mathrm{g}> \\
& \mathrm{CP}_{3}=<-\mathrm{a} \rightarrow-\mathrm{b} \rightarrow+\mathrm{c},+\mathrm{a} \rightarrow+\mathrm{e},+\mathrm{b} \rightarrow+\mathrm{c}> \\
& \mathrm{CP}_{4}=<-\mathrm{a} \rightarrow-\mathrm{b} \rightarrow-\mathrm{c} \rightarrow+\mathrm{f},+\mathrm{a} \rightarrow+\mathrm{d},+\mathrm{b} \rightarrow-\mathrm{c} \rightarrow+\mathrm{g}> \\
& \mathrm{CP}_{5}=<-\mathrm{a} \rightarrow-\mathrm{b} \rightarrow+\mathrm{c} \rightarrow-\mathrm{d} \rightarrow+\mathrm{f},+\mathrm{a} \rightarrow+\mathrm{e},+\mathrm{b} \rightarrow-\mathrm{c} \rightarrow+\mathrm{g}> \\
& \mathrm{CP}_{6}=<-\mathrm{a} \rightarrow-\mathrm{b} \rightarrow-\mathrm{c} \rightarrow+\mathrm{f},+\mathrm{a} \rightarrow+\mathrm{d},+\mathrm{b} \rightarrow+\mathrm{c} \rightarrow-\mathrm{e} \rightarrow+\mathrm{g}>
\end{aligned}
$$

The $\mathrm{CP}$ such as $\mathrm{CP}_{3}, \mathrm{CP}_{4}, \mathrm{CP}_{5}$ and $\mathrm{CP}_{6}$ begins with the initial state but may end in a different state in case of error such as deadlock and unspecified reception.

Let us consider test sequence generation for the following Concurrent Path:

$\mathrm{CP}_{2}=<-\mathrm{a} \rightarrow-\mathrm{b} \rightarrow-\mathrm{c} \rightarrow+\mathrm{f},+\mathrm{a} \rightarrow+\mathrm{e} \rightarrow-\mathrm{f} \rightarrow-\mathrm{g},+\mathrm{b} \rightarrow+\mathrm{c} \rightarrow-\mathrm{e} \rightarrow+\mathrm{g}>$.

There are two kinds of constraints imposed by causal relationships among the events in a CP: (1) ordering imposed by each sequential FSM and (2) ordering imposed by matching send and receive events. In $\mathrm{CP}_{2}$, we have the following constraints of the first kind:

- $-\mathrm{a} \rightarrow-\mathrm{b} \rightarrow-\mathrm{c} \rightarrow+\mathrm{f}$,

- $+\mathrm{a} \rightarrow+\mathrm{e} \rightarrow-\mathrm{f} \rightarrow-\mathrm{g}$, and

- $+\mathrm{b} \rightarrow+\mathrm{c} \rightarrow-\mathrm{e} \rightarrow+\mathrm{g}$. 
The set of constraints of the second kind in $\mathrm{CP}_{2}$ is:

- $\{-\mathrm{a} \rightarrow+\mathrm{a},-\mathrm{b} \rightarrow+\mathrm{b},-\mathrm{c} \rightarrow+\mathrm{c},-\mathrm{e} \rightarrow+\mathrm{e},-\mathrm{f} \rightarrow+\mathrm{f},-\mathrm{g} \rightarrow+\mathrm{g}\}$.

If we apply reachability analysis to $\mathrm{CP}_{2}$ enumerating all possible interleavings, we get 36 event sequences for testing. To alleviate this state space explosion, we construct a minimal length time-event sequence which represents the above causal relationships.

Definition 4: A Minimal Causality Path (MCP) corresponding to a $\mathrm{CP}$ is a minimal length Global Event sequence.

To compute Minimal Causality Paths, first calculate the linear logical time of events in a CP, then obtain GEs for each logical time by collecting all events having the same logical time. The MCP is the sequence of GEs aligned in increasing order of logical time.

If we suffix the events in MCPs with the FSM identifier, then

$$
\mathrm{MCP}_{2}=-\mathrm{a} \rightarrow(-\mathrm{b},+\mathrm{a}) \rightarrow\left(-\mathrm{c}_{1},+\mathrm{b}\right) \rightarrow+\mathrm{c}_{3} \rightarrow-\mathrm{e} \rightarrow+\mathrm{e} \rightarrow-\mathrm{f} \rightarrow(+\mathrm{f},-\mathrm{g}) \rightarrow+\mathrm{g} .
$$

The notation $(-b,+a)$ means the two events occur concurrently, i.e., they are mutually independent. Therefore, the order of occurrences between the two events does not matter. The MCPs obtained in this way constitute concise and accurate test sequences for concurrent systems when the duration of events can be abstracted to unit time.

\section{MODEL ENHANCEMENT}

If the duration of events cannot all be set to unit time, the reduced test sequence can lead to lower verdict capability. When the trace of an event sequence conforms to a Global Event Sequence, we may conclude that the system conforms to specifications. However, when the trace does not conform, the result is inconclusive. To investigate the verdict capability quantitatively for a given GE sequence, we propose the following measure.

Definition 5 : The Verdict Capability (VC) of a test sequence or a GE sequence $\left(\mathrm{GES}_{\mathrm{k}}\right)$ for a Concurrent Path $\mathrm{CP}_{\mathrm{k}}$ is defined as follows:

$$
\mathrm{VC}=|\mathrm{EX}(\mathrm{GESk})| / \mathrm{INTk} \text {, }
$$

where INTk is the number of paths in the reachability tree of CPk and $\mathrm{EX}(\mathrm{GESk})$ is the set of all interleaved event sequences that can be obtained 
from $\mathrm{GES}_{k}$ by juxtaposing the GEs in $\mathrm{GES}_{k}$ for all possible ordering. The function $|E|$ of a set $E$ is the cardinality of $E$.

Note that Definition 5 can also be used to compute the VC for MCPs because MCP is a special case of GES. For example, the VC for $\mathrm{MCP}_{2}$ is $8 / 36$, because the number of paths in the reachability tree of $\mathrm{CP}_{2}$ is 36 , and the number of interleaved event sequences that can be derived from $\mathrm{CP}_{2}$ is 8 . Thus, there is a high possibility of getting inconclusive verdict if we test the system in Figure 1 with respect to $\mathrm{MCP}_{2}$. Therefore, it is necessary to devise a test sequence generation method with the desired verdict capability without incurring state space explosion.

So far, the value of $d$ in the logical clocks for representing the duration of an operation in Section 3.1 is assumed to be unit time. For test sequence generation, if $d$ may assume any natural number or any real number in a certain range, the verdict capability can be increased and the model will be more useful. In this section, we shall consider test sequence generation when the value of $d$ of each event takes on a natural number between 1 and $d_{m}$. Common matrix notation is used in the following description.

Definition 6: For a concurrent system, the Concurrent Path $k$ (CP), the Duration (D) and the Logical times (L) of the j-th event in $\mathrm{CP}_{\mathrm{k}}$ for FSM $i$ are denoted as $\mathrm{CP}_{k}[i][j], \mathrm{D}_{k}[\mathrm{i}][\mathrm{j}]$ and $\mathrm{L}_{\mathrm{k}}[\mathrm{i}][\mathrm{j}]$ respectively.

If the duration of each event in a $\mathrm{CP}$ is known, the corresponding Global Event sequence can be constructed in the same way used for MCPs except that the duration values are not necessarily 1's. The Global Event Sequence (GES) can be viewed as a test sequence for the given concurrent system. Note that a MCP is a special type of GES in which all event durations are one unit long.

Take $\mathrm{CP}_{2}$ in Section 3.2 as an example. According to Definition $6, \mathrm{D}_{2}$ (the unit time) and $\mathrm{L}_{2}$ (corresponding to $\mathrm{MCP}_{2}$ in Section 3.2) are represented as follows:

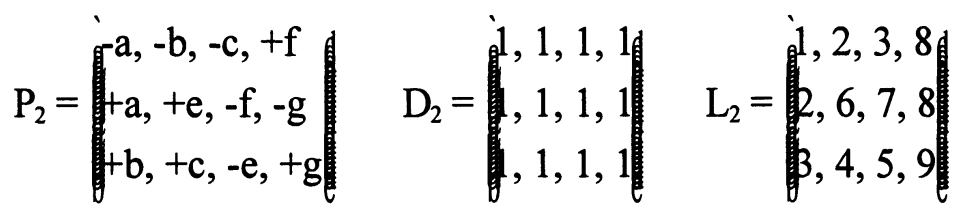

$\mathrm{L}_{2}$ is obtained from $\mathrm{CP}_{2}$ and $\mathrm{D}_{2}$ by the method described in Section 3.2. Because there are three 2-tuples representing concurrent events in $\mathrm{L}_{2}, \mathrm{MCP}_{2}$ covers eight $(=2 ! \times 2 ! \times 2 !)$ paths in the reachability tree of $\mathrm{CP}_{2}$.

To generate the test sequence (or equivalently to generate GES for combinations of relaxed event durations), we can assign event durations $D_{k}$ to 
the desired values. However, in the remainder of this section, for simplicity of illustration, we shall consider the case when the duration of each event in $\mathrm{CP}_{2}$ is either 1 or 2 only.

Example 1: Given $\mathrm{CP}_{2}$ and $\mathrm{D}_{2}^{\prime}$, we calculate $\mathrm{L}_{2}^{\prime}$ and the corresponding $\mathrm{GES}_{2}{ }^{\prime}$ as follows:

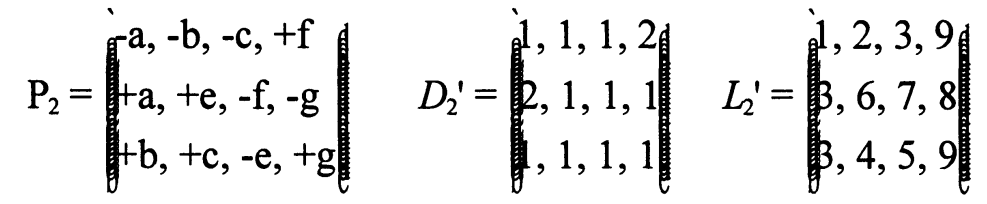

$$
\begin{aligned}
& \mathrm{GES}_{2}{ }^{\prime}=-\mathrm{a} \rightarrow-\mathrm{b} \rightarrow(-\mathrm{c},+\mathrm{a},+\mathrm{b}) \rightarrow+\mathrm{c} \rightarrow-\mathrm{e} \rightarrow+\mathrm{e} \rightarrow-\mathrm{f} \rightarrow-\mathrm{g} \rightarrow(+\mathrm{g},+\mathrm{f}) .
\end{aligned}
$$

The values in $\mathrm{L}_{2}^{\prime}$ represent the linear logical times of the corresponding events. Note the numbers ' 3 ' and ' 9 ' in $\mathrm{L}_{2}$ '. The events in $\mathrm{CP}_{2}$ having the same value given in $\mathrm{L}_{2}^{\prime}$ (e.g. -c, $+\mathrm{a}$, and $+\mathrm{b}$ ) are concurrent. Thus, the GES covers twelve $\left(=3 ! \times 2\right.$ !) paths in the reachability tree of $\mathrm{CP}_{2}$. This example shows how the verdict capability can be improved by relaxing the event durations.

If all cases of event durations in $\mathrm{CP}_{2}$ (which may be either 1 or 2) are considered, there are a total of $4096\left(=2^{12}\right)$ different combinations of event durations for the 12 events. In general, given the maximum duration $d_{m}$ and number of events $M$, there are $d_{m}{ }^{M}$ combinations to be considered. Because the number of combinations can become very large, tremendous amount of computation is needed if we calculate GESs for all the combinations. Thus, it is necessary to reduce the amount of computation by introducing some constraints.

First we define concurrent event and non-concurrent event as follows: An event $\mathrm{e}$ in a CP is said to be a concurrent event if there exists at least one other event $\mathrm{e}^{\prime}$ in $\mathrm{CP}$ such that $\mathrm{e} \| \mathrm{e}^{\prime}$. Otherwise $\mathrm{e}$ is a non-concurrent event.

Property 1: The position (i.e. sequential order) of a non-concurrent event in a GE sequence obtained from a $\mathrm{CP}$ is independent of the event duration of the other events.

Proof: The proof is straightforward from the fact that there is a causality relation between a non-concurrent event and every other event.

As a result of Property 1, we do not need to consider the event duration of non-concurrent events because they do not change the order of a GE sequence in any way. 
Example 2: The events '-a', '+e', and '-f' in CP2 are non-concurrent and the other events are concurrent. Therefore, the number of combinations of event duration we need to consider is reduced to $512(=29)$ for the 9 concurrent events in CP2 instead of $4096(=212)$.

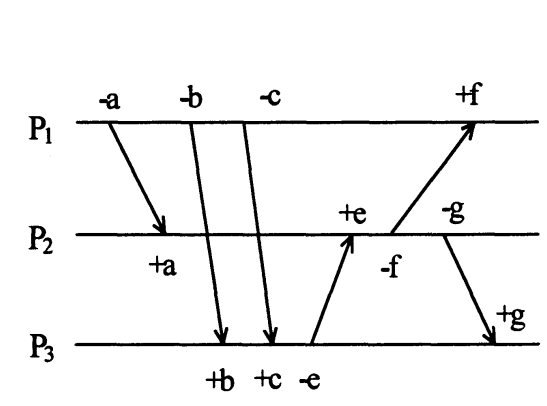

(a) Acomputation: $\mathrm{CP}_{2}$

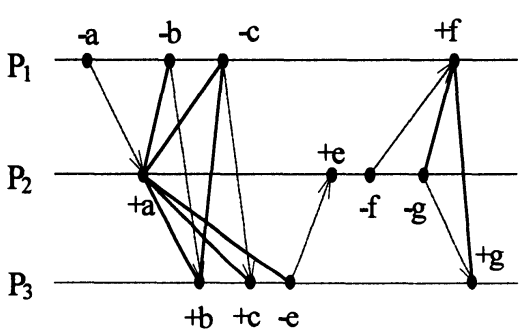

(b) The corresponding conarrency graph

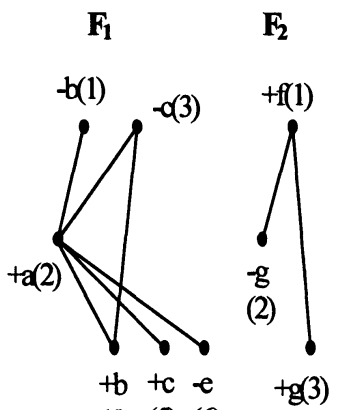

(4) (5) (6)

(c) Depth-first search trees

$\mathbf{F}_{1} \quad \mathbf{F}_{2}$

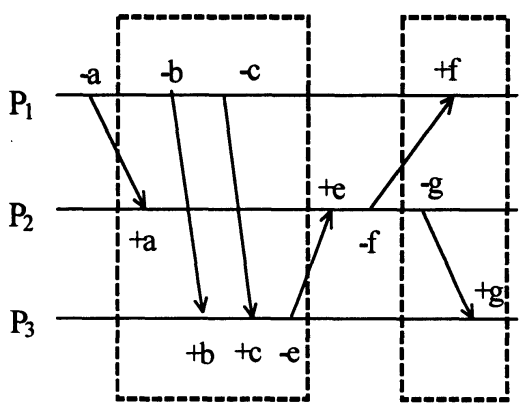

(d) CESs of $\mathrm{CP}_{2}$

Figure 2. Example of CES construction

In order to reduce the number of combinations of event duration further, we introduce the concept of Connected Event Set (CES):

\section{Definition 7: Connected Event Set (CES)}

Given a CP, a concurrency graph can be constructed consisting of vertices corresponding to events in the $\mathrm{CP}$ and edges joining all pairs of vertices whose corresponding events are concurrent. Generally a concurrency graph has one or more disjointed subgraphs. A Connected Event Set is the set of all events in a maximal connected subgraph of a concurrency graph. 
We shall illustrate Definition 7 with the examples in Figure 2. Given $\mathrm{CP}_{2}$ shown in Figure 2(a), we obtain the concurrency graph in Figure 2(b) using the method described in Section 3.1. A solid dark line between two events means the two events are concurrent. Note that the concurrent relation is symmetric and non-transitive. From Figure 2(b), we can obtain the CESs shown in Figure 2(d). A CES may be constructed using an efficient search technique instead of enumerating all combinations of events. In the example, we used the depth-first search technique as shown in Figure 2(c) where the numbers in parentheses denote the order of searching.

A property of CES is given below:

Property 2: For a CP with CESs $F_{1}, F_{2}, \ldots, F_{n}$, given $F_{i}$ and $F_{j}$ such that $1 \leq i$, $\mathrm{j} \leq \mathrm{n}$, and $\mathrm{i} \neq \mathrm{j}$, the following hold:

2.1: If $e \rightarrow f \quad\left(\right.$ or $f \rightarrow e$ ) for $f \in F_{i}, e \in C P$ and $e \notin F_{i}$, then $e \rightarrow f^{\prime}\left(\right.$ or $f^{\prime} \rightarrow e$ ) for any $f \in F_{i}$. In other words, $e \rightarrow F_{i}\left(\right.$ or $F_{i} \rightarrow e$ ).

2.2: If $f_{i} \rightarrow f_{j}$ for $f_{i} \in F_{i}, f_{j} \in F_{j}$, then $f_{i}^{\prime} \rightarrow f_{j}^{\prime}$ for any $f_{i}^{\prime} \in F_{i}$ and $f_{j}^{\prime} \in F_{j}$. In other words, $\quad F_{i} \rightarrow F_{j}$.

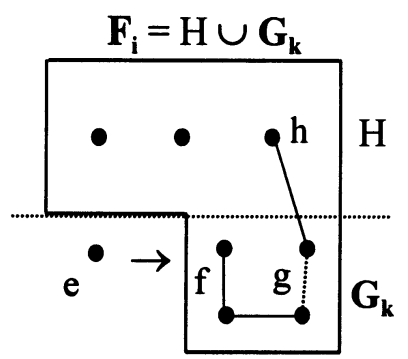

(a) Diagram illustrating Property 2.1

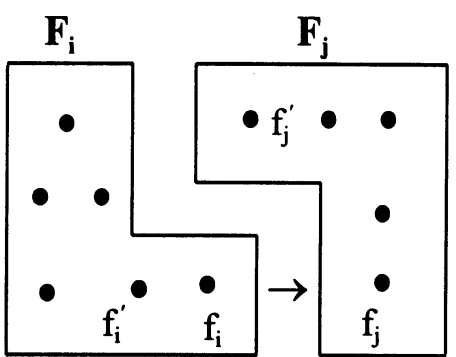

(b) Diagram illustrating

Property 2.2

Figure 3. Diagrams showing properties of CES

Proof : 2.1 With reference to Figure 3(a), if the cardinality of $F_{i}$ is $N$ and $G_{k}$ is a subset of $F_{i}$ with $k$ elements, then $G_{N}$ is equal to $F_{i}$. Assume $e \rightarrow f$ holds for $e \notin F_{i}$ and $f \in F_{i}$. First, we set $G_{1}$ to $\{f\}$, then $e \rightarrow G_{1}$. Assume we have a set $G_{k}$ satisfying e $\rightarrow G_{k}$ for $1 \leq k \leq N-1$. It is sufficient to prove that we can find a set $G_{k+1}$ satisfying $e \rightarrow G_{k+1}$ from the known sets $G_{k}$ and $F_{i}$.

Let set $H$ be the set $F_{i}-G_{k}$. Since $G_{k}$ and $H$ together make up the set $F_{i}$, there exists at least one concurrent relation, say $g \| h$, between the two events $g \in G_{k}$ and $h \in H$. Let us construct $G_{k+1}$ by including $h$ in 
$G_{k}$, i.e. $G_{k} \cup\{h\}$. Since e $\notin F_{i}$, either e $\rightarrow h$ or $h \rightarrow$ e hold. If we assume $h \rightarrow e$, since $e \rightarrow g$ then $h \rightarrow g$. Since this contradicts with $g \|$ $h, e \rightarrow h$ must hold. Thus $\mathrm{e} \rightarrow \mathrm{G}_{\mathrm{k}+1}$.

The proof of the converse can be obtained in a similar manner.

2.2 Refer to Figure 3(b). Assume $f_{i} \rightarrow f_{j}$ holds for $f_{i} \in F_{i}$ and $f_{j} \in F_{j}$. Then by Property $2.1, f_{i} \rightarrow F_{j}$ holds. This in turn implies $F_{i} \rightarrow f_{j}^{\prime}$ for any $f_{j}{ }^{\prime} \in F_{j}$. Thus $F_{i} \rightarrow F_{j}$.

Example 3: Consider $\mathrm{CP}_{2}$ in Section 3.2. According to Property 2 and by applying the method given in Section 3.1, we can obtain the constraint $F_{1} \rightarrow$ $\mathrm{F}_{2}$, where $\mathrm{F}_{1}=\{+\mathrm{a},-\mathrm{b},+\mathrm{b},-\mathrm{c},+\mathrm{c},-\mathrm{e}\}$ and $\mathrm{F}_{2}=\{+\mathrm{f},-\mathrm{g},+\mathrm{g}\}$ as shown in Figure 2. Hence, the order in any $\mathrm{GE}$ sequence for $\mathrm{CP}_{2}$ can be represented as follows:

$$
\mathrm{GES}_{2}=-\mathrm{a} \rightarrow(+\mathrm{a},-\mathrm{b},+\mathrm{b},-\mathrm{c},+\mathrm{c},-\mathrm{e}) \rightarrow+\mathrm{e} \rightarrow-\mathrm{f} \rightarrow(+\mathrm{f},-\mathrm{g},+\mathrm{g}) .
$$

Note that the above GES consists of a sequential event and a CES in sequential order. In other words, concurrent behavior may exhibit some sequential characteristics in the high-level abstraction.

Definition 8: A linear logical base time, $T_{B}$, for a given event is defined as $T_{B}$ $\equiv \max \left(c_{i}\right.$, ts $)$ for a receive event, and $T_{B} \equiv c_{i}$ for a send event, where $c_{i}$ is the logical time of the preceding event associated with FSM $\mathrm{P}_{\mathrm{i}}$ and $t s$ is the timestamp of the receive event as defined in Section 3.1.

Three cases of a leading event (i.e., the first event of a sequence in chronological order) in each FSM in a CES F are of interest:

- case 1: a receive event in $\mathrm{F}$ corresponding to a send event which occurred before $\mathrm{F}$,

- case 2: a send event, and

- case 3: a receive event corresponding to a send event in F.

The following property states that the order of an event sequence and the difference in logical time between events are dependent on the event duration of the events in $\mathrm{F}$ but independent of the event duration of the events in the other CESs and any non-concurrent events.

Property 3: The base times $\left(T_{B}\right)$ for the leading events in cases 1 and 2 in a $\mathrm{CES} F$ are the same and equal to the maximum linear logical time, $\mathrm{T}_{\max }$, among all events that occurred before $F$.

Proof : Let $e$ be a leading event in case 1 or 2 in $F$, and let $T_{e}, T_{B}$ and $\delta$ be the logical time, base time, and duration of the event $e$, respectively. Then $T_{e}$ can be determined as follows:

$$
\mathrm{T}_{\mathrm{e}}=\mathrm{T}_{\mathrm{B}}+\delta \text {. }
$$


Since the base time of $e$ is determined prior to $\mathrm{F}$, the relation $\mathrm{T}_{\mathrm{B}} \leq \mathrm{T}_{\max }$ must hold. If we assume $T_{B} \neq T_{\max }, T_{e}$ can be represented as follows:

$$
\mathrm{T}_{\mathrm{e}}=\mathrm{T}_{\max }-\varepsilon+\delta, \quad \varepsilon=\mathrm{T}_{\max }-\mathrm{T}_{\mathrm{B}} .
$$

As $\varepsilon>0$, if we set $\delta$ such that $0<\delta \leq \varepsilon$, then $\mathrm{T}_{\max }-\varepsilon<\mathrm{T}_{\mathrm{e}} \leq \mathrm{T}_{\max }$ holds. This contradicts Property 2 which indicates $T_{e}>T_{\max }$. Thus $T_{B}=T_{\max }$.

According to Property 3, given a CES F, the base times of events belonging to cases 1 and 2 are equivalent to the corresponding $T_{\max }$. Furthermore, the base times of events belonging to case 3 are determined by the logical time of the matching send events in F. Therefore, the events before $\mathrm{F}$ do not influence the order of event sequence or the difference in logical time between events in $F$. This means the event sequence and the time difference between events with $T_{B}=0$ are equivalent to those with $T_{B}=T_{\max }$, thus enabling independent derivation of GE sequences in each CES.

Example 4: In $F_{2}$ of Example 3, ' $-\mathrm{g}$ ' is a case 2 event, ' $+\mathrm{f}$ ' is a case 1 event and ' $+g$ ' is a case 3 event. The base times of events ' $-g$ ' and ' $+f$ ' are equal to the logical time $\left(=T_{\max }\right)$ of the event ' $-\mathrm{f}$ ', and the base time of ' $+\mathrm{g}$ ' is equal to the logical time of the event ' $\mathrm{g}$ '.

Definition 9: Given a CES, a partial-GES (denoted as pGES) is defined as a sequence of GEs containing all events in the CES.

Note that there may be one or more pGESs for a given CES. Based on the results of Properties 1,2 and 3, the following algorithm is developed to generate GE sequences for a concurrent system.

Algorithm 1: (Global Event Sequence generation for a concurrent system) Step 1. Generate CESs for a given CP. Let these CESs be $F_{1}, F_{2}, \ldots, F_{n}$. Step 2. For $\mathrm{i}=1,2, \ldots, \mathrm{n}$, do:

Generate $p$ GESs from $F_{i}$ with the initial value of $T_{B}$ set to 0 for each combination of event duration. Let the output be $B_{i}=\left\{\right.$ pGES $_{i}{ }^{1}$, pGES $_{i}{ }^{2}$, $\ldots$, pGES $\left._{\mathrm{i}} \mathrm{p}^{(\mathrm{i})}\right\}$, where $\mathrm{pGES}_{\mathrm{i}}^{\mathrm{k}}$ is the $\mathrm{k}$-th pGES of $\mathrm{F}_{\mathrm{i}}$, and $\mathrm{p}(\mathrm{i})$ is the total number of pGESs.

Step 3. Obtain GESs by applying the Cartesian product $B_{1} \times B_{2} \times \ldots \times B_{n}$ and inserting non-concurrent events into the predetermined positions.

Example 5: Let us apply Algorithm 1 to $\mathrm{GES}_{2}$ in Example 3. Since $\left|\mathrm{F}_{1}\right|=6$ and $\left|F_{2}\right|=3,72\left(=2^{6}+2^{3}\right)$ cases of event durations are considered in Step 2. As a result, 10 and 4 partial-GESs are obtained for $\mathrm{CES} \mathrm{F}_{1}$ and $\mathrm{F}_{2}$, respectively. The GE sequence generated in Step 3 is given by: 
$\{-\mathrm{a}\} \times \mathrm{B} 1 \times\{+\mathrm{e}\} \times\{-\mathrm{f}\} \times \mathrm{B} 2$

$=\{-\mathrm{a}\} \times\left\{\mathrm{pGES}_{1}{ }^{1}, \operatorname{pGES}_{1}{ }^{2}, \ldots, \operatorname{pGES}_{1}{ }^{10}\right\} \times\{+\mathrm{e}\} \times\{-\mathrm{f}\} \times\left\{\mathrm{pGES}_{2}{ }^{1}, \mathrm{pGES}_{2}{ }^{2}\right.$, $\left.\ldots, \mathrm{pGES}_{2}{ }^{4}\right\}$

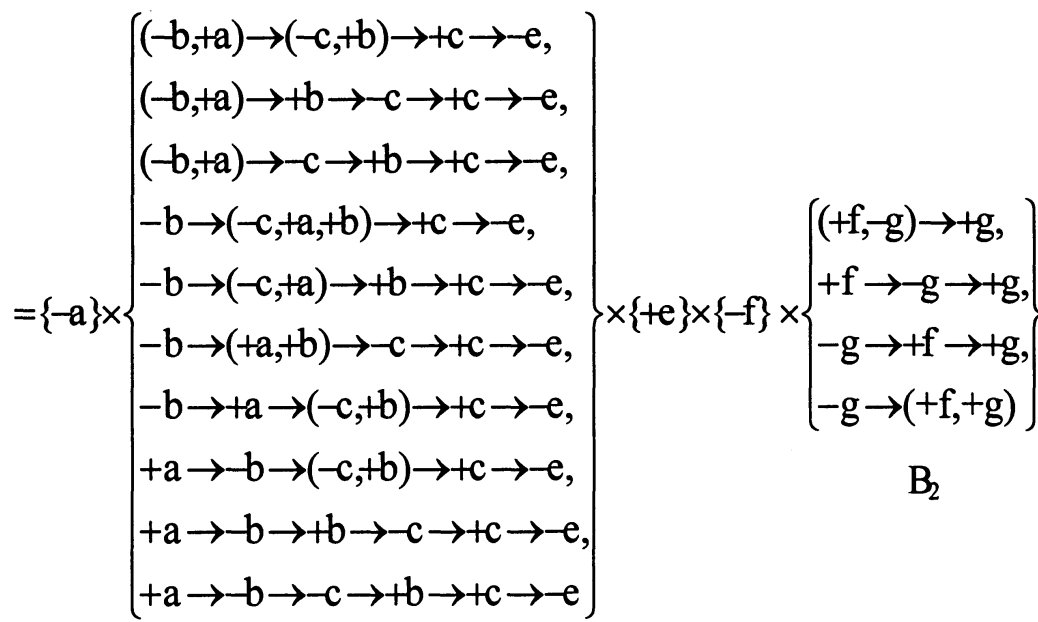

$\mathrm{B}_{1}$

Step 2 of Algorithm 1 provides a saving in computation complexity from $2^{\{|\mathrm{F}||+\ldots+| \mathrm{Fn} \mid\}}$ to $2^{|\mathrm{F}|}+\ldots+2^{|\mathrm{Fn}|}$, where 2 is the number of different event durations and $\left|F_{i}\right|$ is the number of events in CES $F_{i}$. The savings are partially offset by Steps 1 and 3 of Algorithm 1 if a CES exists. However, the computation overhead is small compared to the saving in Step 2. If there are $\mathrm{k}$ different event durations, the computation saving is $\mathrm{k}^{\{|\mathrm{F}|+\ldots+|\mathrm{Fn}|\}}$ minus $\mathrm{k}^{|\mathrm{F}|}+\ldots$ $+\mathrm{k}^{|\mathrm{Fn}|}$.

In Example 5, $72\left(=2^{6}+2^{3}\right)$ cases of event durations are considered in Step 2 in order to generate $\mathrm{GE}$ sequences for $\mathrm{CP}_{2}$. If all the combinations of event durations in $\mathrm{CP}_{2}$ which are either 1 or 2 are considered, there will be 4096 cases. In the same fashion, we need only 72 out of 4096 cases for $\mathrm{CP}_{1}, 72$ out of 4096 for $\mathrm{CP}_{2}, 8$ out of 16 for $\mathrm{CP}_{3}, 32$ out of 64 for $\mathrm{CP}_{4}, 64$ out of 128 for $\mathrm{CP}_{5}$, and 64 out of 128 cases for $\mathrm{CP}_{6}$. In total, 312 cases of event durations are sufficient to generate GE sequences for all CPs where the total number of combinations of event durations in all the CPs is 8528 .

It can be easily shown that the $\mathrm{VC}$ of test sequences obtained for $\mathrm{CP}_{2}$ in Example 5 is $24 / 36$ whereas the $\mathrm{VC}$ of $\mathrm{MCP}_{2}$ is $2 / 9$. This represents an improvement of nearly three times. Recall the maximum value of $\mathrm{VC}$ is 1 . To attain VC values closer to 1 , a larger maximum duration value $d_{m}$ is needed. Therefore, there is a tradeoff between the amount of computation required for test sequence generation and the resultant verdict capability. 


\section{GENERALIZATION OF THE MODEL}

In this section, we discuss how the models proposed in Section 4 can be further generalized by :

i) a technique to use real value of ' $d$ ' by the logical clocks for representing the duration of an operation, and

ii) application of the model to timed automata (used for describing the timing constraints in real-time systems).

Consider $\mathrm{CP}_{2}$ in Section 3.2. Note that events '-b', '-c', and '-g' happened before events ' $+b$ ', ' $+c$ ', and ' $+g$ ' respectively. The notation $(x, y, z)$ means that events $\mathrm{x}, \mathrm{y}$ and $\mathrm{z}$ occurred concurrently. As a result of Property 2 in Section 4, the generalized GE Sequence (GGES) corresponding to $\mathrm{CP}_{2}$ is as follows:

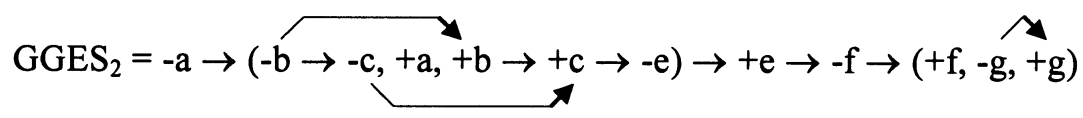

In this formula, all the causal relations between events are represented and the duration of each event can be assigned any arbitrary value. If each event takes one unit time, $\mathrm{GGES}_{2}$ becomes $\mathrm{MCP}_{2}$ in Section 3.2 and if each event takes one or two unit time, GGES becomes $\mathrm{GES}_{2}$ in Section 4. In this section, we shall derive GGESs corresponding to CPs as follows:

$$
\begin{aligned}
& \operatorname{GGES}_{1}=-\mathrm{a} \rightarrow(-\mathrm{b} \rightarrow+\mathrm{c} \rightarrow-\mathrm{d},+\mathrm{a},+\mathrm{b} \rightarrow-\mathrm{c}) \\
& \mathrm{GGES}_{3}=-\mathrm{a} \rightarrow(-\mathrm{b},+\mathrm{a}, \mathrm{d}) \\
& \mathrm{GGES}_{4}=-\mathrm{a} \rightarrow(-\mathrm{b} \rightarrow-\mathrm{d},+\mathrm{f},+\mathrm{d} \rightarrow-\mathrm{f},-\mathrm{g},+\mathrm{g}) \\
& \mathrm{GGES}_{5}=-\mathrm{a} \rightarrow(-\mathrm{b} \rightarrow+\mathrm{c} \rightarrow-\mathrm{d},+\mathrm{a},+\mathrm{b} \rightarrow-\mathrm{c}) \\
& \text { GGES }_{6}=-\mathrm{a} \rightarrow(-\mathrm{b} \rightarrow-\mathrm{c},+\mathrm{a},+\mathrm{b} \rightarrow+\mathrm{c} \rightarrow-\mathrm{d},)
\end{aligned}
$$

By merging GGES's while preserving the causal relation among events, the Combined GGES in Figure 4 is obtained (the algorithm is omitted here, see [Kim 98] for details) ${ }^{1}$. An event is labeled at an arrow head. A circular arc

1. $\mathrm{GGES}_{4}, \mathrm{GGES}_{5}$ and $\mathrm{GGES}_{6}$ are not shown in the figure for simplicity. 
crossing a number of arrows (e.g., arc A just after event '-a') is used to indicate that the events after event ' $-a$ ' and before event ' $+e$ ' (i.e., $-b,-c,+a$, $+b,+c,-e)$ are in a Connected Event Set (CES) (refer to Figure 2). Both $\mathrm{GGES}_{3}$ and GGES $\mathrm{S}_{1}$ are branched off from events in the CES of GGES 2 . Also, there may be causal relationship among the events of different FSMs in a CES (for example, events ' $b$ ' and ' $+b$ ' just after arc A).

The merged (or combined) GGES analysis shown above has advantages over the conventional reachability tree analysis for the following reasons:

- timing constraints can be described because the duration of each event and the causal relation among the events are provided, and

- concurrent behavior can be represented.

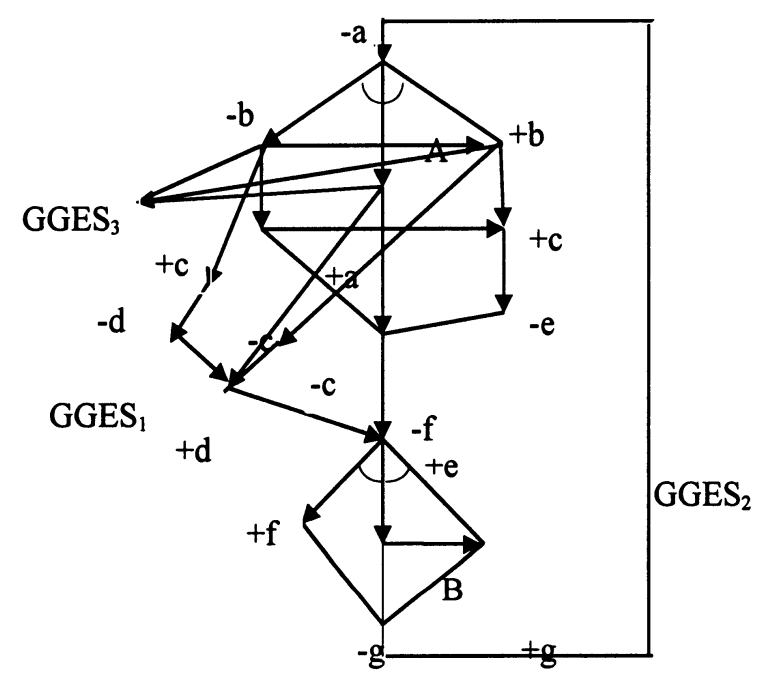

Figure 4. Combined GGES of the concurrent systems in Figure 3

The absence of these properties is a major contributor to the state space explosion problem in conventional reachability analysis as mentioned in Section 1.

Given a sequential sequence of events, $\mathrm{e}^{1} \rightarrow \ldots \rightarrow \mathrm{e}^{\mathrm{i}} \rightarrow \ldots \rightarrow \mathrm{e}^{\mathrm{n}}$ where $\mathrm{n}$ is an integer, let us define ' $T$ ' Furthermore, let $t_{i}$ be the time distance between $\mathrm{e}^{\mathrm{i}}$ and the previous event, $\mathrm{e}^{\mathrm{i}-1}$. $t_{i}$ may take on a range of time values, say low $w_{i} \leq t_{i} \leq$ high $_{i}$, where low $_{i}$ and $\mathrm{high}_{\mathrm{i}}$ are any real numbers. In other words, $\mathrm{e}^{\mathrm{i}}$ occurs after $\mathrm{e}^{\mathrm{i}-1}$ within a time range between $\operatorname{low}_{i}$ and $h_{i g h}$. Now if $T_{i}$ is measured from the initial event until the occurrence of the $\mathrm{i}$-th event (including $\mathrm{t}_{\mathrm{i}}$ ), the timing constraints on an event are: 
1. For an event, $\mathrm{e}^{\mathrm{n}}$, having a single directed path from the initial event, time $\mathrm{T}_{\mathrm{n}}$ is in the range: $\sum_{\mathrm{i}=1}{ }^{\mathrm{n}} \operatorname{low}_{\mathrm{i}} \leq \mathrm{T}_{\mathrm{n}} \leq \sum_{\mathrm{i}=1}{ }^{\mathrm{n}}$ high $_{\mathrm{i}}$.

2. For an event, $\mathrm{e}^{\mathrm{n}}$ with more than one (say $\mathrm{K}$ ) directed paths from the initial event, time $T_{n}$ is: $\operatorname{MAX}\left(\sum_{\mathrm{i}=1}{ }^{\mathrm{m}} \operatorname{low}_{1, \mathrm{i}}, \ldots, \sum_{\mathrm{j}=1}{ }^{\mathrm{n}} \operatorname{low}_{\mathrm{K}, \mathrm{j}}\right) \leq \mathrm{T}_{\mathrm{n}} \leq \mathrm{MIN}\left(\sum_{\mathrm{i}=1}{ }^{\mathrm{m}}\right.$ high $_{1, \mathrm{i}}, \ldots, \sum_{\mathrm{j}=1}{ }^{\mathrm{n}}$ high $_{\mathrm{K}, \mathrm{j}}$ ) where $\sum_{\mathrm{i}=1}{ }^{\mathrm{m}} \operatorname{low}_{1, \mathrm{i}} \leq \mathrm{T}_{\mathrm{n}}{ }^{1} \leq \sum_{\mathrm{i}=1}{ }^{\mathrm{m}} \operatorname{high}_{1, \mathrm{i}}, \ldots, \sum_{\mathrm{j}=1}{ }^{\mathrm{n}}$ low $_{K, j} \leq T_{n}{ }^{K} \leq \sum_{j=1}{ }^{n}$ high $_{K, j}$ and $i, j, m, n$, and $K$ are integers. $T_{n}{ }^{k}$ is the logical time for the k-th path to complete execution.

For example, for an event sequence: $-a \rightarrow-b \rightarrow-c$, the time $T_{-c}$ for event '-c' is low $_{-a}+\operatorname{low}_{-b}+\operatorname{low}_{-c} \leq T_{-c} \leq \operatorname{high}_{-a}+$ high $_{-b}+$ high $_{-c}$ because there is a directed path. Also $\mathrm{T}_{\mathrm{tc}}$ is: $\operatorname{MAX}\left(\left(\operatorname{low}_{-\mathrm{a}}+\mathrm{low}_{\mathrm{b}}+\mathrm{low}_{-\mathrm{c}}+\mathrm{low}_{+\mathrm{c}}\right),\left(\mathrm{low}_{-\mathrm{a}}+\mathrm{low}_{-}\right.\right.$ $\left.\left.{ }_{b}+\operatorname{low}_{+b}+\operatorname{low}_{+c}\right)\right) \leq T_{+c} \leq \operatorname{MIN}\left(\left(\right.\right.$ high $_{-a}+$ high $_{-b}+$ high $_{-c}+$ high $\left._{+c}\right),\left(\right.$ high $_{-a}+$ high $_{-b}+$ high $_{+b}+$ high $\left._{+c}\right)$ ) because there are two paths from -a to $+c$, i.e., $-a \rightarrow$ $-\mathrm{b} \rightarrow-\mathrm{c} \rightarrow+\mathrm{c}$ and $-\mathrm{a} \rightarrow-\mathrm{b} \rightarrow+\mathrm{b} \rightarrow+\mathrm{c}$. Because the timing constraints are specified by using the above rule after the generation of GGES, it may not be possible for some events to happen because of conflicts in the timing constraints.

By using the Combined GGES, tests can be generated dynamically. This means a test can be performed without a predetermined scenario specified with time schedules. Instead, when an event is observed, we can execute an appropriate operation to generate events satisfying the causality and timing constraints in the Combined GGES. If an observed event satisfies the constraints, the verdict is 'pass'. If not, the verdict is 'fail'. Note that sometimes it may not be possible to generate an event due to errors such as deadlocks or conflicts in the timing constraints.

\section{CONCLUSIONS}

In order to test and verify the concurrent behaviors of protocols running in a concurrent system, traditional approaches map multiple modules into a single module and analyze the sequential behavior of the resultant monolithic module. Other approaches apply interleaving methods for events in a concurrent system. Recently some attempts have been made to adopt a causality based method or a true concurrency model in protocol verification and testing of concurrent systems (e.g., [Probs 90, Ulric 95, Kim 96, Henni 97, Ulric 97].

In this paper, we have enhanced our previous model [Kim 96] by relaxing the assumption on logical time from unit time to any natural or real numbers in describing timing constraints. An efficient algorithm for deriving test cases for the relaxed event duration has been proposed and evaluated in terms of 
Verdict Capability and computational efficiency. We have showed that the saving in testing effort can be quite substantial. We believe our model forms a sound foundation for further investigation in this area.

\section{REFERENCES}

[Araka 91] Arakawa, N. and Sonecoka, T., "A Test Case Generation Method for Concurrent Programs," Proceedings of the 4th Int'l Workshop on Protocol Test Systems, pp. III 35 - 47, Leidschendam, The Netherlands.

[Caval 93] Cavalli, A., Kim, S. U., and Maigron, P., "Improving Conformance Testing for LOTOS," Proceedings of the Sixth Int'l Conference on Formal Description Techniques, pp. 371 - 386, Boston, USA, Oct.

[Henni 97] Henniger O., "On Test Case Generation from Asynchronously Communicating State Machines," Testing of Communicating Systems Vol 10 (also this is 10th Int't Workshop on Testing of Communicating Systems, Cheju Island, Korea) edited by M. Kim, S. Kang and et al., Chapman \& Hall, pp. 255 - 271, Sept.

[ISO 94] ISO IS 9646 OSI Conformance Testing Methodology and Framework.

[Itoh 83] Itoh, M. and Ichikawa, H., "Protocol verification using reduced reachability analysis," The Trans. of the IECE of Japan, Vol. 66, No. 2, pp. 88 - 93, Feb.

[Kajiw 85] Kajiwara, M., Ichikawa, H., Itoh, M., and Yoshida, Y., "Specification and Verification of Switching Software," IEEE Tr. on Communications, Vol. COM-33, No. 3, pp. 193 - 198, March.

[Kim 96] Kim, M. C., Chanson, S. T., Kang S. and Shin J.W. "An Approach for testing asynchronous communicating systems," Testing of Communicating Systems (also this is 9th Int't Workshop on Testing of Communicating Systems, Darmstadt, Germany) edited by B. Baumgarten and et al. pp. 141 - 154, Sept.

[Kim 98] Kim, M. C., Shin J.W., Chanson, S. T., and Kang S. "An Approach for testing asynchronous communicating systems," Technical Report, School of Information and Computer Engineering, Information and Communications Univ., Daejon, Korea

[Lampo 78] Lamport, L., "Time, Clocks, and the Ordering of Events in a Distributed System," $C A C M$, Vol.21, No. 7, pp. 558 - 565, July.

[Lee 93] Lee, D., Sabnani, K. K., Kristol, D. M. and Paul, S., "Conformance Testing of Protocols Specified as Communicating FSMs," IEEE INFOCOM, pp. 115 - 127.

[Luo 94] Luo, G., Bochmann, G. V., and Petrenko, A., "Test Selection Based on Communicating Nondeterministic Finite-State Machines Using a Generalized WpMethod," IEEE Tr. on Software Engineering, Vol. 20, No. 2, pp. 149 - 161.

[Probs 90] Probst, D. K. and Li, H. F., "Using Partial-order Semantics to Avoid the State Explosion Problem in Asynchronous Systems," 2nd Int'l Conference on Computer-aided Verification, pp. 146 - 155.

[Rayna 92] Raynal, M., “About Logical Clocks for Distributed Systems," ACM Operating Systems Review, Vol. 26, No. 1, pp. 41 - 48, Jan.

[Taylo 92] Taylor, R. N., Levine, D. L., and et al., "Structural Testing of Concurrent Programs," IEEE Tr. on Software Engineering, Vol. 18, Iss.3, pp. 206 - 215, March.

[Tai 95] Tai, K. C. and Carver, R. H., "Testing of Distributed Programs," Handbook of Parallel and Distributed Computing, ed. A. Zomaya, McGraw Hill.

[Ulric 95] Ulrich, A. and Chanson, S. T., "An Approach to Testing Distributed Software Systems," 15th Int'l Symposium on Protocol Specifications, Testing, and Verification, pp. $121-136$. 
[Ulric 97] Ulrich, A. and Koenig H., "Specification-based testing of concurrent systems," Int'l Con. On Formal Description Techniques and Protocol Specifications, Testing, and Verification, pp. 7 - 22, Osaka, Japan, Nov.

[West 78] West, C. H., "General Technique for Communications Protocol Validation," IBM J. RES. DEVELOP, Vol. 22, No. 4, pp. 393 - 404, July.

[Young 88] Young, M., and Taylor, R. N., "Combining Static Concurrency Analysis with Symbolic Execution," IEEE Tr. on Software Engineering, Vol. 14, No. 10, pp. 1499 - 1511 , Oct. 